\title{
Laser Tattoo Removal, Precautions, and Unwanted Effects
}

\author{
Yvonne Eklund • Agneta Troilius Rubin \\ Centre for Laser and Vascular Anomalies, Department of Dermatology, Skane University Hospital, \\ Malmö, Sweden
}

\begin{abstract}
Laser tattoo removal uses the physical properties of photoselective thermolysis in order to remove tattoo pigment. The technique has gradually improved over the years with the development of Q-switched lasers, with overall good results and a relatively low degree of adverse effects. However, lasers cannot always erase the unwanted tattoo completely, and there are still risks of unwanted effects such as scarring, pigment changes, ink darkening, and potential aggravation of latent skin conditions. This chapter will discuss the precautions that have to be taken and what pitfalls to avoid before starting the procedure of laser tattoo removal.
\end{abstract}

(C) 2015 S. Karger AG, Basel

\section{Introduction}

Tattoos have been part of human culture for thousands of years, with a wide range of patterns and symbolic values. Earlier in the history of mankind, tattooing was not as mainstream a feature as it has become today, with millions of people having tattoos or planning on getting one. The quest for expressing your individual identity seems to be central. However, many tattoo customers have regrets about their tattoo sooner or later in life, and this leads to an increasing world- wide demand for effective and safe ways of tattoo removal [1].

Since 1993, at our hospital department, we have removed different kinds of tattoos, mainly with a quality-switched, or Q-switched (QS), pulsed laser. In order to qualify for our laser treatment regime, patients have to fulfil certain criteria since financing of the sessions is subsidised with public means. The tattoos removed have varied, including stigmatising symbols and patterns (fig. 1), tattoos associated with an earlier criminal life, and medical tattoos. Additionally, some patients, and especially women, have had tattoos forced upon them by a dominant partner. Cosmetic tattoos with extreme disfiguration have also been accepted for treatment. Furthermore, all patients with traumatic tattoos have been offered laser treatment, as well as those who have developed different allergic reactions to the tattoo ink.

\section{Early Tattoo Removal and Non-Specific Wavelength Lasers}

Over the years, different chemical abrasions and surgical techniques have been used for tattoo removal, but with many side effects, and especially scarring and pigment retention. Heating and 

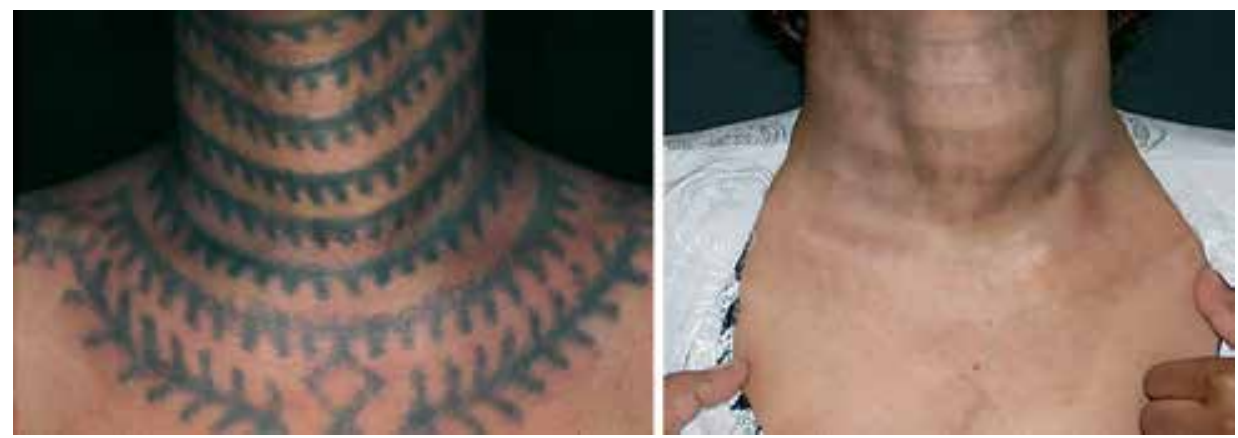

Fig. 1. Shamanistic tattoo for medical effect of goitre. Skin type V. Before and after several QS $\mathrm{Nd}$ :YAG laser 1,064 $\mathrm{nm}$ treatments. Postinflammatory hyperpigmentation to a certain degree on the neck where it was treated only 3 times. Photos: Dr A Troilius Rubin.

burning the skin in order to get rid of tattoo pigment have been used for centuries, including using fire, charcoal, and cigarettes. In the 1980s, removing tattoos in a more controlled manner, with heat, was tried with an infrared coagulator. Still, scarring was common due to the non-specific heat damage of dermal tissue [2]. Try-outs with lasers started, such as with argon lasers, but since the wavelengths were non-specific for the tattoo ink and instead largely absorbed by melanin and haemoglobin, the procedure often ended up with the same problem as earlier, causing scarring. Another alternative at this time was the carbon-dioxide la$\operatorname{ser}\left(\mathrm{CO}_{2}\right.$ laser $)$, with vaporisation of the upper skin layers as its major mechanism in order to clear away the tattoo ink by transepidermal migration. However, there were great differences in depth during one and the same session, which often resulted in thick scarring and residual pigment [3].

\section{Principles of Q-Switched Laser in Tattoo Removal}

Anderson and Parrish's principle of selective photothermolysis in 1983 revolutionised the treatment of tattoos. They proposed that if a wavelength was well absorbed by the target and the pulse width was equal to or shorter than the tar- get's thermal relaxation time, the heat generated from the laser would be confined to the target [4]. Considering that ink particles are very small and will hold heat in just a fraction of a second, one must use extremely short pulses. With the introduction of so-called QS lasers in the 1990s, with specific wavelengths that were well absorbed by ink particles and with a pulse duration of only nanoseconds, there was a golden standard for laser tattoo removal. Increasing evidence has also shown that there seems to be an important mechanism of photo-acoustic waves created by heat differences in the skin, contributing to the effect [5].

The most common colour of a tattoo is black, and following that are blue, green, red, yellow, and orange. All of these colours have different wavelength absorption, ranging from about 420 to 800 $\mathrm{nm}$ (table 1). A suitable laser with a specific wavelength is chosen according to this principle (table 2). However, nowadays, especially in professional tattoos, there is a wide range of colour mixes, making it sometimes a challenge to foresee the pigment reaction to a chosen laser wavelength. This problem is particularly seen in so-called cover-up tattoos placed on an earlier tattoo.

If the right wavelength and adjustable parameters such as spot size and fluence are chosen, the heat accumulation in the target colour will result in fragmentation of the ink, with the surrounding 
Table 1. Absorption spectrum of the different tattoo pigment colours, measured in nanometres

\begin{tabular}{ll}
\hline Colour & Maximum absorption, $\mathrm{nm}$ \\
\hline Black & $600-800^{*}$ \\
Blue-green & $656-808$ \\
Permanent green & $570-800$ \\
Red-violet & $500-570$ \\
Orange & $420-540$ \\
Yellow & $470-485$ \\
\hline
\end{tabular}

* Black absorbs over the entire spectrum, with no specific peak (adapted from Verhaeghe 2010 [6]).

Table 2. QS lasers with their specific wavelengths and colour targets

\begin{tabular}{cll}
\hline $\begin{array}{l}\text { Wavelength, } \\
\mathrm{nm}\end{array}$ & Laser & Colours \\
\hline 532 & Nd:YAG laser & $\begin{array}{l}\text { Red, orange, } \\
\text { (brown, yellow) } \\
\text { Black, blue, green, } \\
\text { (purple, brown) } \\
\text { Black, blue, green, } \\
\text { (brown) }\end{array}$ \\
754 & Ruby laser & Alexandrite laser, blue, (brown) \\
\hline 1,064 & Nd:YAG laser & Black \\
\hline
\end{tabular}

The colours in brackets indicate some but less satisfactory absorption than for the other chromophores (adapted from Verhaeghe 2010 [6] and Karsai and Raulin 2011 [21]).

tissue spared [6]. Immediately after the laser treatment, an expected whitening of the skin will be seen, and this is usually a sign of good light absorption by the ink. The reaction represents dermal and epidermal vacuolisation due to localised steam formation and is often accompanied by normal pinpoint bleeding to a moderate degree, as a sign of slight epidermal disruption. The skin will heal within the next 2-7 days. In order to avoid bacterial infection, we recommend application of antiseptic ointment or dressing during this period. In the weeks following the laser procedure, there will be a gradual clearing of the tattoo pigment by immune cells, such as macrophages and lymphocytes, via the lymph nodes. However, where the ink goes after the lymph nodes is not yet established, which means that a full comprehension of all immunological pathways involved is still lacking. Therefore, there have been questions about the safety of the ink particles regarding their potential toxicity, both before and after laser treatment, and their allergenic and carcinogenic properties $[7,8]$. These fears have made a few physicians quit removing tattoos with lasers.

\section{Initial Consultation}

The patient should be thoroughly informed about what laser treatment of a tattoo actually comprises. There is a common misconception among the public that it is easy to remove a tattoo. Multiple laser treatments are always required, and many times, it is not possible to remove the tattoo completely, especially for multilayered, re-tattooed ones. It is important that the patient receives information on this concept and that an estimation of the number of laser sessions needed is made at the initial consultation. Otherwise, there will be a risk of poor treatment adherence, with the patient underestimating the effort and time required to clear a tattoo. In 2009, the Kirby-Desai scale was presented as a practical tool for assessing the number of laser sessions needed. In short, a darker skin type; dense tattoo ink; professional, complex tattoos with many colours; and distal tattoos on the extremities will require many laser treatments [9]. In some cases, up to 20 sessions or more are needed. Amateur tattoos are usually easier to remove, with fewer numbers of treatments, because of the irregularity of the depths of the pigment and the lower amount of pigment used.

In a substantial percentage of patients, there will be some residual pigment resisting further laser removal, depending on the factors mentioned above. It might be tempting to be more aggressive in treatment parameters or to use shorter treatment 
intervals, but since tattoo ink is cleared by dermal macrophage cells several weeks after each laser procedure, it is of utmost importance that there is a treatment interval of at least 6-8 weeks. Shorter treatment intervals have been reported to interfere with immune cell activity and may even increase the risk of scarring and ink retention [10]. If the tattoo has many features that will make full clearance impossible, it may be wiser to discourage the patient from any laser treatment at all. In order to avoid disagreement over the result or other issues, photos should be taken before starting the laser sessions and also during the treatment period.

\section{Medical Evaluation}

Because of the theoretical systemic spread of fragmented ink particles after laser treatment, we never treat pregnant or lactating women. Additionally, patients with severe diabetes are discouraged from QS laser treatment due to their diminished wound healing and higher risk of complicated infections. With regard to the safety of personnel, human immunodeficiency virus or hepatitis could be a risk factor. However, to our knowledge, no cases of patient-laser surgeon transmission have yet been reported. Furthermore, some medications might interfere with a successful result, such as retinoids, which are reported to increase the potential for scarring in some cases, although other reports do not fully support this [11]. Earlier gold injections against arthritis can result in hyperpigmentation (chrysiasis), even if the gold was given years ago [12]. It is also of value to know if the patient has any allergies, considering that it sometimes will be necessary to orally or topically treat the patient with medication. Questioning about earlier scarring is important since this can reveal whether the patient has a tendency to form hypertrophic scars or keloids. If an increased risk of scarring is suspected, information should be given, and as a precaution, a corticosteroid ointment and silicon dressing could be applied after each laser procedure.

\section{General Precautions}

Even though the rates of adverse effects are low with QS lasers, there are still some general risks that have to be considered before starting laser treatment. For example, a laser machine is not safe to use if the treating personnel does not have the proper education and experience. Even if photoselective thermolysis is the goal of tattoo removal, the wrong choice of wavelength, fluence, or pulse width will not reach this goal and will instead cause dermal damage and long-term side effects. Parameter settings that work well and safely in one patient might be wrong in another due to differences in skin texture and pigmentation. There are also risks of treating a tattoo with too many pulses on a single occasion since it can lead to a too-intense tissue reaction. In one rare case, this reaction led to a compartment syndrome of the forearm. For this reason, newer methods (such as the R20 method) that comprise multiple passes on the same occasion for better tattoo clearing should, according to our opinion, perhaps not be performed at distal parts of extremities $[13,14]$. Even in the hands of an experienced laser surgeon, it is sometimes best to perform treatment first on a small test surface, especially if there are uncertainties about the right choice of wavelength and the response of the ink particles.

\section{Dermatological Assessment}

There is a necessity of having professional skills in the medical field since there are dermatological reactions, nevi, and tumours found within a tattoo in some cases. This needs to be evaluated before treatment. For example, psoriasis, lichen, and vitiligo can be exacerbated by tattooing (Koebner phenomenon) and potentially also by the procedure of lasing. Other histological reactions in tattoos might be reactions to the pigment itself, and these can be granulomatous or lichenoid, and 
sometimes even pseudolymphomas might develop [15]. All of this can be a diagnostic challenge. Additionally, different tumours can occur within a tattoo, such as basalioma, squamous cell carcinoma, and malignant melanoma. Whether this is a coincidence or actually caused by the tattoo is not established. A general rule is to never put a tattoo over a pigmented lesion and, before tattoo removal, to always excide a nevus or tumour in order to acquire a histopathological diagnosis $[16,17]$. Finally, infectious complications should be avoided, and if there are signs of an active skin infection, whether bacterial or viral, laser treatment should be postponed and the infection treated. Additionally, if the tattoo is near an area with inactive intermittent herpes infection, e.g. the mouth, one should consider herpes prophylaxis in order to avoid reactivation.

\section{Scarring}

The risk of developing scars from laser tattoo removal is generally reported to be low, or just a few percent [18]. However, using too-high energy fluence and a too-large spot size increases the risk of deeper thermal injury, with potential scarring as a consequence. If exaggerated inflammation postlaser is noticed, a cooling device can be used, together with the application of a potent corticosteroid ointment. In a reported series of treatmentresistant tattoos, as many as $18.8-25 \%$ developed scarring, probably due to more intense treatment [16]. If there is a need to penetrate deeper, a larger spot size should be tried first, instead of increasing the fluence, in order to get less light scattering in the skin and hence less damage [19]. However, there are risks even with this strategy. Although the right parameters might be used, with an adequate tissue reaction, it is well established that with multiple laser sessions, the risk of fibrosis as well as hypopigmentation or postinflammatory hyperpigmentation will gradually increase [10].

\section{Dyspigmentation}

Hyper- and hypopigmentation as adverse effects of laser tattoo removal are more common than scarring [20]. Hyperpigmentation might follow inflammation or numerous laser treatments. It is more common in darker skin with more melanin and can often develop even after an adequate laser reaction. Hyperpigmentation can often be managed with longer intervals, letting the pigment gradually fade. It can often be a benefit to prescribe a bleaching agent, such as hydroquinone $2-4 \%$. In all cases, ultra-violet exposure should be avoided. The risk of hypopigmentation will increase not only with too-high fluences and subsequent inflammation but also if shorter wavelengths are used since this means potential competing absorption by the melanosomes in the basal layer of the epidermis. Consequently, in order to avoid hypopigmentation in dark-skinned patients, longer wavelengths and lower fluences are preferable. If hypopigmentation is seen, longer treatment intervals would be of value so that the skin and its melanosomes have time to recover. Most of the time, loss of pigment is transient, but it may persist for several months or years. However, some authors report permanent loss of melanin pigment in up to $10 \%$ of all cases [21].

\section{Ink Retention and Paradoxical Ink Darkening}

Black and blue pigments are often the easiest tattoo pigments to remove, while moderate success is seen with green. A poorer outcome is specifically seen with white, purple, turquoise, yellow, orange, and flesh-toned colours. Sometimes, instead of a QS laser, a pulsed dye laser $(510 \mathrm{~nm})$ can be used to treat pigment on the red-yellow spectrum, with good results described; however, it is not the first-line choice since its pulse duration is considerably longer $[21,22]$.

Paradoxical ink darkening might occur after QS laser treatment, especially in tattoos containing 

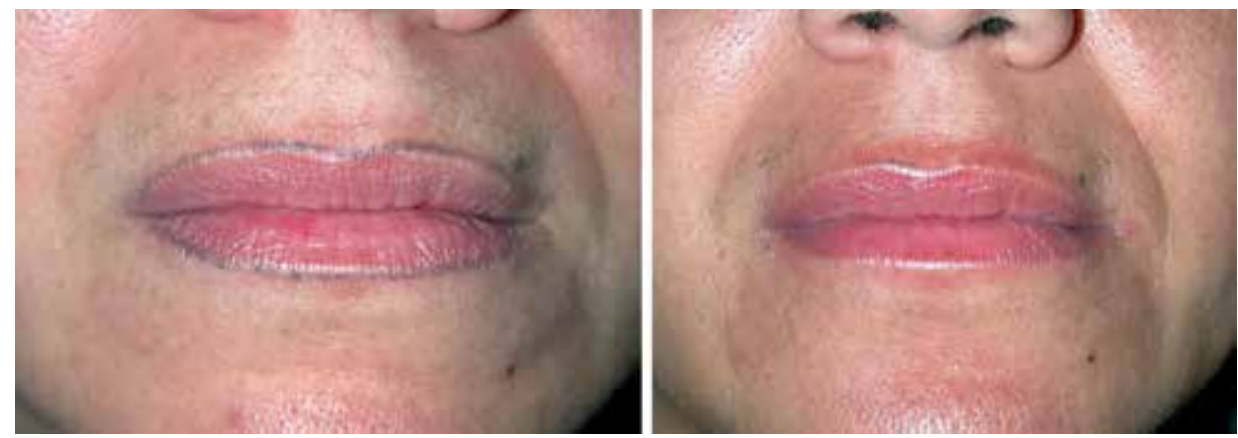

Fig. 2. Paradoxical ink darkening to the left. Treated successfully with QS Nd:YAG laser 1,064 nm to the right. Photos: Dr A Troilius Rubin.

titanium dioxide, such as white ink, which is often used to add brilliance to other tattoo inks. Ink darkening might also develop due to the ferric oxide in flesh-toned cosmetic tattoos, often used as permanent make-up. The mechanism is probably due to a chemical reduction of the pigment with the laser light [23]. A trial session would be valuable in such cases since this ink darkening may be irreversible. After the immediate whitening is gone, about 10-20 min post-treatment, an evaluation of a possible colour change can be made. Even if a chemical reduction does occur, according to our experience and other reports, switching to a QS laser with an appropriate wavelength will often remove the darkened pigment (fig. 2) [24, 25]. Some authors suggest using a $\mathrm{CO}_{2}$ laser in treating flesh-toned cosmetic tattoos or altered ink as a good alternative, taking advantage of the mechanism of transepidermal pigment migration [26]. In some cosmetic tattoos, such as permanent eyeliner, it is quite common to see fanning of the ink downwards, into the lower eyelid. This is usually easy to remove with a QS laser and does not need more than 1-2 treatments.

\section{Traumatic Tattoos}

Traumatic tattoos are the result of accidents in which pigmented particles get embedded in the skin. These can arise from abrasive damage, e.g. asphalt from a bicycle accident, or a penetrating force, such as a graphite pencil. Unfortunately, a quite common cause of traumatic tattoos is explosions from fireworks and gun powder (fig. 3). This situation constitutes a specific risk since the material can explode during laser treatment and create pitted scars in the skin of the patient [27]. Therefore, these cases should always be treated with caution. Success in clearing the traumatic tattoo will depend on the depth and on the particle size. Deeply embedded material may not be erased completely, and the patient should be informed of this. Harder materials, e.g. asphalt and car paint, will need more treatments, while softer materials, e.g. dirt and mascara, need less [28]. If the particles are too large, the laser will not be sufficient to target them with its short pulses. In such situations, an ablative laser, such as an Erbium:YAG or $\mathrm{CO}_{2}$ laser, could be beneficial [29].

\section{Allergic Reactions}

If the patient has an allergy to the tattoo, this will appear as a typical allergic dermatitis or as either a granulomatous or a lichenoid reaction within specific colour areas [15]. In our experience, most common is an allergic reaction to red pigment, but an allergy to all sorts of colours can develop, 

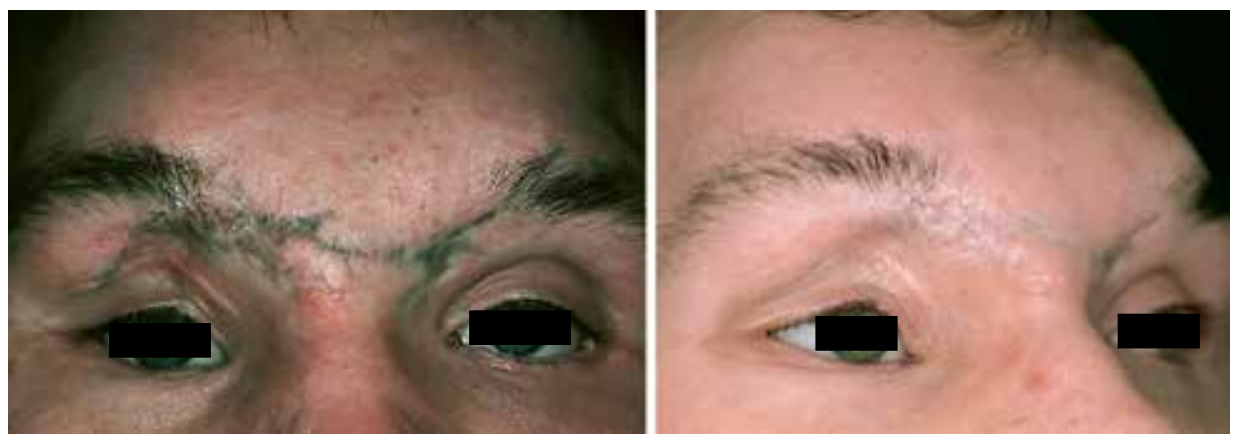

Fig. 3. Traumatic tattoo due to gun powder. Six sessions of QS Nd:YAG laser 1,064 nm. Photos: Dr A Troilius Rubin.
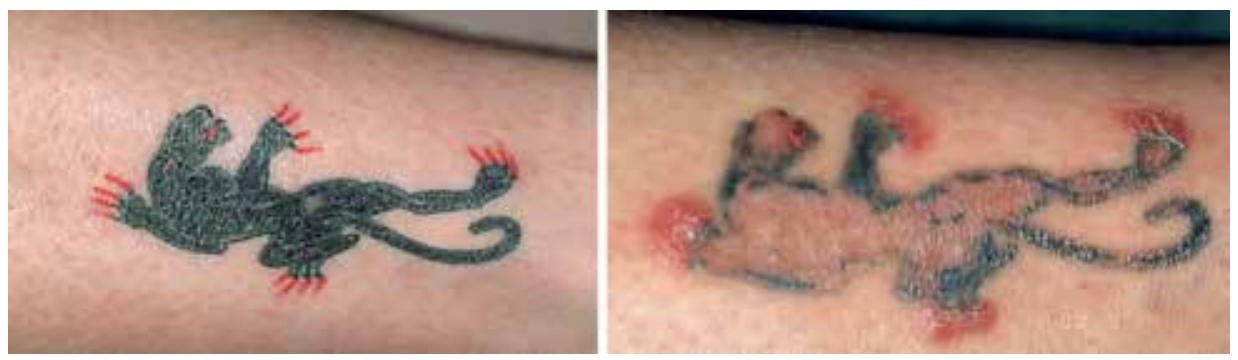

Fig. 4. Allergic reaction to red tattoo ink probably induced by the laser after two sessions of QS $\mathrm{Nd}$ YYAG laser $532 \mathrm{~nm}$. Photos: Dr A Troilius Rubin.

with the least common being to black. Sometimes, it takes a long time, up to a year or so, to develop an allergy. This is due to chemical changes in the ink over time, with sunlight being an important factor. Laser treatment itself can also, in rare cases, induce allergy to the fragmented ink (fig. 4) [30, 31]. As mentioned earlier, after QS laser treatment, tattoo ink is cleared by cells that transport the ink fragments via the lymphatic system. Consequently, there is a potential risk of a systemic reaction to the ink particles after laser treatment, especially if there has been a local allergic reaction. Being prepared for such a reaction is therefore essential. The reports on clinical reactions following laser treatment have so far been mainly composed of mild or moderate allergic dermatitis, although generalised reactions have also been described in some published cases [32]. To avoid a systemic reaction, some laser surgeons recommend a $\mathrm{CO}_{2}$ or Erbium:YAG laser instead of, or before, QS laser treatment [33]. However, in our department, we have successfully treated at least ten patients with a localised allergic reaction to tattoo ink with a QS Nd:YAG laser. Several patients received intralesional corticosteroids or were prescribed daily application of a potent corticosteroid ointment in order to suppress most of the localised allergic reaction 1-2 months before laser treatment. At the time of treatment, all patients received an oral antihistamine one hour before laser treatment and were also kept under close surveillance during and 4 hours after the session. So far, there have not been any systemic allergic reactions during or after the procedure. 


\section{Newer Treatments and Future}

Today, there are already promising studies and ongoing usage of the next generation of QS lasers, using pulses in the range of picoseconds, which are reported to create even better results in clearing a tattoo. This is attributed to the shortening of the pulse, matching the thermal relaxation time of the tattoo ink better and generating more effective photo-acoustic waves [5, 34]. However, these new machines are currently very costly, and it is too early to tell if they are worthwhile for their improved capacity for clearing tattoo ink. Hence, more studies are needed.

Newer techniques are combinations of already-known modalities, such as pretreating the skin with a fractional $\mathrm{CO}_{2}$ laser or an Erbium:YAG laser and then directly treating the tattoo with a QS laser [35]. Another combination is the fractional QS laser (which is also new) with a QS laser with a full beam $[5,36]$. Furthermore, in order to lessen the degree of scattering and to increase the penetration of light, it is possible to use diascopy (compression of the skin with a glass plate) during the QS laser session. In our experience, this seems to lead to a better effect of clearing deeper pigment. There are also reports on transdermal application of glycerol for the same effect [37].
For the future, one of the most important things is the development of safer tattoo pigment. Considering the large number of tattooed people who want to remove a tattoo, it would be so much easier if the pigment were made of nontoxic, biodegradable particles. However, this means that a tattoo will not 'be for life' and will therefore lose its symbolic value for many tattoo admirers. Should permanent ink be the overall choice of the tattoo community, it would be of great advantage if this ink had well-known absorption wavelengths, thereby making it easier to remove with a QS laser. Five years ago, a new tattoo ink was marketed in the United States, designed as microbeads of polymethylmethacrylate containing bioresorbable ink with specific laser wavelengths (InfinitInk; Freedom, Inc., N.J., USA). We will hopefully see more of this and of such initiatives in the future. In the meantime, the next important step is the development of lasers. For example, it would be of help to have lasers that can emit a large variety of wavelengths for treating all of the different ink colours that exist today while, at the same time, being able to penetrate deeper. Even if we do achieve all of the above improvements that are wished for, it would be very favourable if the public were given better information on the limitations of laser tattoo removal before getting a tattoo.

\section{References}

1 Laumann AE, Derick AJ: Tattoos and body piercings in the United States: a national data set. J Am Acad Dermatol 2006;55:413-421

2 Colver GB: The infrared coagulator in dermatology. Dermatol Clin 1989;7: 155-167.

3 Kent KM, Graber EM: Laser tattoo removal: a review. Dermatol Surg 2012;38 $1-13$.

4 Anderson RR, Parrish JA: Selective photothermolysis: precise microsurgery by selective absorption of pulsed radiation. Science 1983;220:524-527.

\footnotetext{
5 Luebberding S, Alexiades-Armenakas M: New tattoo approaches in dermatology. Dermatol Clin 2014;32:91-96.

6 Verhaeghe E: Chapter 7: Techniques and Devices Used for Tattoo Removal; in De Cuyper C, Pérez-Cotapos ML (eds): Dermatologic Complications with Body Art: Tattoos, Piercings and Permanent Make-Up. Heidelberg, Springer-Verlag, 2010, pp 91-105.

7 Ferguson JE, Andrew SM, Jones CJ, August PJ: The Q-switched neodymium: YAG laser and tattoos: a microscopic analysis of laser-tattoo interactions. Br J Dermatol 1997;137:405-410.
}

${ }_{8}$ Bäumler W, Eibler ET, Hohenleutner U, Sens B, Sauer J, Landthaler M: Q-switch laser and tattoo pigments: first results of the chemical and photophysical analysis of 41 compounds. Lasers Surg Med 2000;26:13-21.

$>9$ Kirby W, Desai A, Desai T, Kartono F, Geeta P: The Kirby-Desai Scale: a proposed scale to assess tattoo-removal treatments. J Clin Aesthet Dermatol 2009;2:32-37. 
10 Kirby W, Chen CL, Desai A, Desai T: Causes and recommendations for unanticipated ink retention following tattoo removal treatment. J Clin Aesthet Dermatol 2013;6:27-31.

11 Alissa A: Concomitant use of laser and isotretinoin, how safe. Grapevine, American Society for Laser Medicine and Surgery, 2011.

12 Almoallim H, Klinkhoff AV, Arthur AB, Rivers JK, Chalmers A: Laser induced chrysiasis: disfiguring hyperpigmentation following Q-switched laser therapy in a woman previously treated with gold. J Rheumatol 2006;33:620-621.

13 Rheingold LM, Fater MC, Courtiss EH: Compartment syndrome of the upper extremity following cutaneous laser surgery. Plast Reconstr Surg 1997;99:14181420.

14 Kossida T, Rigopoulos D, Katsambas A, Anderson RR: Optimal tattoo removal in a single laser session based on the method of repeated exposures. J Am Acad Dermatol 2012;66:271-277.

15 Bassi A, Campolmi P, Cannarozzo G, Conti R, Bruscino N, Gola M, Ermini S, Massi D, Moretti S: Tattoo-associated skin reaction: the importance of an early diagnosis and proper treatment. Biomed Res Int 2014;2014:354608.

16 Karsai S, Krieger G, Raulin C: Tattoo removal by non-professionals - medical and forensic considerations. J Eur Acad Dermatol Venereol 2010;24:756-762.

17 Pohl L, Kaiser K, Raulin C: Pitfalls and recommendations in cases of laser removal of decorative tattoos with pigmented lesions: case report and review of the literature. JAMA Dermatol 2013; 149:1087-1089.

18 Bernstein EF: Laser treatment of tattoos. Clin Dermatol 2006;24:43-55.

19 Choudhary S, Elsaie ML, Leiva A, Nouri K: Lasers for tattoo removal: a review. Lasers Med Sci 2010;25:619-627.
20 Klein A, Rittmann I, Hiller KA, Landthaler M, Bäumler W: An Internetbased survey on characteristics of laser tattoo removal and associated side effects. Lasers Med Sci 2014;29:729-738.

21 Karsai S, Raulin C: Laser treatment of tattoos and other dyschromia; in Raulin C, Karsai S (eds): Laser and IPL Technology in Dermatology and Aesthetic Medicine. Berlin/Heidelberg, Springer Verlag, 2011, pp 189-210.

22 Stafford TJ, Tan OT: 510-nm pulsed dye laser and alexandrite crystal laser for the treatment of pigmented lesions and tattoos. Clin Dermatol 1995;13:69-73.

23 Kirby W, Kaur RR, Desai A: Paradoxical darkening and removal of pink tattoo ink. J Cosmet Dermatol 2010;9:149-151.

24 De Cuyper C: Permanent makeup: indications and complications. Clin Dermatol 2008;26:30-34.

25 Fitzpatrick RE, Lupton JR: Successful treatment of treatment-resistant laserinduced pigment darkening of a cosmetic tattoo. Lasers Surg Med 2000;27:358361.

26 Mafong EA, Kauvar AN, Geronemus RG: Surgical pearl: removal of cosmetic lip-liner tattoo with the pulsed carbon dioxide laser. J Am Acad Dermatol 2003; 48:271-272.

27 Taylor CR: Laser ignition of traumatically embedded firework debris. Lasers Surg Med 1998;22:157-158.

28 Troilius A: Effective treatment of traumatic tattoos with a Q-switched Nd:YAG laser. Lasers Surg Med 1998;22: 103-108.

29 Kunzi-Rapp K, Krähn GM, Wortmann S, Peter RU: Early treatment of traumatic tattoo by erbium-YAG laser. Br J Dermatol 2001;144:219-221.
30 Serup J, Hutton Carlsen K: Patch test study of 90 patients with tattoo reactions: Negative outcome of allergy patch test to baseline batteries and culprit inks suggests allergen(s) are generated in the skin through haptenization. Contact Dermatitis 2014;71:255-263.

31 Vasold R, Naarmann N, Ulrich H, Fischer D, König B, Landthaler M, Bäumler W: Tattoo pigments are cleaved by laser light-the chemical analysis in vitro provide evidence for hazardous compounds. Photochem Photobiol 2004;80: 185-190.

32 Yorulmaz A, Onan DT, Artuz F, Gunes R: A case of generalized allergic contact dermatitis after laser tattoo removal. Cutan Ocul Toxicol, DOI: 10.3109/ 15569527.2014.933972.

33 Ibrahimi OA, Syed Z, Sakamoto FH, Avram MM, Anderson RR: Treatment of tattoo allergy with ablative fractional resurfacing: a novel paradigm for tattoo removal. J Am Acad Dermatol 2011;64: 1111-1114.

-34 Brauer JA, Reddy KK, Anolik R, Weiss ET, Karen JK, Hale EK, Brightman LA, Bernstein L, Geronemus RG: Successful and rapid treatment of blue and green tattoo pigment with a novel picosecond laser. Arch Dermatol 2012;148:820-823.

35 Weiss ET, Geronemus RG: Combining fractional resurfacing and Q-switched ruby laser for tattoo removal. Dermatol Surg 2011;37:97-99.

-36 Luebberding S, Alexiades-Armenakas MR: Fractional, nonablative Q-switched 1,064-nm neodymium YAG laser to rejuvenate photoaged skin: a pilot case series. J Drugs Dermatol 2012;11:13001304.

37 McNichols RJ, Fox MA, Gowda A, Tuya S, Bell B, Motamedi M: Temporary dermal scatter reduction: quantitative assessment and implications for improved laser tattoo removal. Lasers Surg Med 2005;36:289-296. 